\title{
Relative Uptake of Minoxidil into Appendages and Stratum Corneum and Permeation through Human Skin In Vitro
}

\author{
JEFFREY E. GRICE, ${ }^{1}$ SUSAN CIOTTI, ${ }^{2}$ NORMAN WEINER, ${ }^{3}$ PETER LOCKWOOD, ${ }^{2}$ \\ SHEREE E. CROSS, ${ }^{1}$ MICHAEL S. ROBERTS ${ }^{1}$ \\ ${ }^{1}$ Therapeutics Research Unit, School of Medicine, The University of Queensland, Princess Alexandra Hospital, Brisbane, \\ Woolloongabba, Qld 4102, Australia \\ ${ }^{2}$ Formerly Pfizer Global Research and Development, Ann Arbor, Michigan \\ ${ }^{3}$ College of Pharmacy, University of Michigan, Ann Arbor, Michigan 48109
}

Received 25 March 2009; revised 8 May 2009; accepted 19 May 2009

Published online 18 June 2009 in Wiley InterScience (www.interscience.wiley.com). DOI 10.1002/jps.21856

\begin{abstract}
We examined uptake of the model therapeutic agent, minoxidil, into appendages, stratum corneum (SC), and through human skin, under the influence of different vehicles. Quantitative estimation of therapeutic drug deposition into all three areas has not previously been reported. Finite doses of minoxidil (2\%, w/v) in formulations containing varying amounts of ethanol, propylene glycol (PG), and water (60:20:20, 80:20:0, and 0:80:20 by volume, respectively) were used. Minoxidil in SC (by tape stripping), appendages (by cyanoacrylate casting), and receptor fluid was determined by liquid scintillation counting. At early times $(30 \mathrm{~min}, 2 \mathrm{~h})$, ethanol-containing formulations (60:20:20 and 80:20:0) caused significantly greater minoxidil retention in SC and appendages, compared to the formulation lacking ethanol (0:80:20). A significant increase in minoxidil receptor penetration occurred with the PG-rich 0:80:20 formulation after $12 \mathrm{~h}$. We showed that deposition of minoxidil into appendages, SC, and skin penetration into receptor fluid were similar in magnitude. Transport by the appendageal route is likely to be a key determinant of hair growth promotion by minoxidil. (c) 2009 Wiley-Liss, Inc. and the American Pharmacists Association J Pharm Sci 99:712-718, 2010

Keywords: absorption; formulation vehicle; membrane transport; passive diffusion; skin; transdermal drug delivery
\end{abstract}

\section{INTRODUCTION}

The differential targeting of topically applied compounds into appendages, stratum corneum (SC) and through skin is an important goal. While

Susan Ciotti's present address is NanoBio Corporation, 2311 Green Road, Suite A, Ann Arbor, MI 48105.

Peter Lockwood's present address is Pfizer Global Research and Development, Groton, CT 06340.

Correspondence to: Michael S. Roberts (Telephone: +61-73240-5803; Fax: +61-7-3240-5806;

E-mail: m.roberts@uq.edu.au)

Journal of Pharmaceutical Sciences, Vol. 99, 712-718 (2010)

(C) 2009 Wiley-Liss, Inc. and the American Pharmacists Association there have been a number of studies examining appendageal deposition, particularly of nanoparticles, ${ }^{1}$ and other work aiming to determine the contribution of appendageal transport to skin penetration, ${ }^{2}$ to our knowledge there are no reports examining the deposition of therapeutic compounds to all three areas. The aim of this work was to study uptake, through a quantitative estimate of deposition of the model therapeutic agent, minoxidil, into appendages, SC, and through human skin, under the influence of different vehicles.

There is increasing recognition of the contributions to transdermal delivery made by "shunt 
pathways" or "appendageal transport," cularly at early times. ${ }^{4}$ This contribution has been evaluated, with varying degrees of acceptance, by indirect methods, for example, by a comparison between areas of different follicular density ${ }^{5}$ or between intact and scarred skin, ${ }^{6}$ by physical blocking of follicular openings, ${ }^{7}$ or by the use of the in vitro skin sandwich technique. ${ }^{8}$ In addition, rather than regarding follicles as shunts for transdermal delivery, some attention has been given to direct targeting of hair follicles by therapeutic agents, such as for acne and alopecia. To this end, attempts have been made to quantitate material retained in follicles after topical application of model penetrants such as dyes ${ }^{9}$ and nanoparticles, ${ }^{1}$ but little has been done with therapeutic compounds and the area remains difficult and incompletely validated. ${ }^{10} \mathrm{~A}$ useful approach is that of differential stripping. ${ }^{11}$ Introduced by Marks and Dawber, ${ }^{12}$ this technique allows quantitation of SC retention by tape stripping and of follicular retention by cyanoacrylate casting. ${ }^{1}$

The targeting of topically applied substances to particular skin sites can be approached by manipulating the components of the formulation. In this work, we chose to prepare formulations with different proportions of three common vehicles, ethanol, propylene glycol (PG), and water. Ethanol and $\mathrm{PG}$ rapidly diffuse into the $\mathrm{SC}^{13}$ and may act as penetration enhancers by a variety of mechanisms. ${ }^{13-15}$ Of interest here are the effects on membrane properties to alter drug permeation, following alcohol diffusion into the membrane. As well, ethanol could potentially extract or solubilize sebum to promote follicular delivery in some cases. The use of water in formulations aids skin hydration, thus enhancing transdermal delivery of many drugs. This may be particularly important when vehicles which tend to desiccate the skin are present, such as ethanol and PG.

While the mechanism of action of the model compound, minoxidil remains unclear, the hair follicle has been regarded as the site of action ${ }^{16}$ and a recent report identified K(ATP) channels responsive to minoxidil located in human follicular dermal papillae. ${ }^{17}$ The uptake of minoxidil was studied by measuring penetration into the receptor of Franz diffusion cells and by differential stripping, consisting of tape stripping (20 times) to sample SC content, which reflects partitioning of minoxidil into the skin barrier layer and cyanoacrylate casting to sample minoxidil deposited within the follicle. Three prototype formulations with differing ethanol, PG, and water content were chosen; in proportion by volume of $60: 20: 20$, 80:20:0 (no water) and 0:80:20 (no ethanol), respectively. Finite doses of $2 \%$ minoxidil $(5 \mu \mathrm{L} /$ $\mathrm{cm}^{2}$ ) were applied to excised full thickness human skin. This dose is similar to that applied to the human scalp under "in use" conditions $(3-5 \mu \mathrm{L} /$ $\mathrm{cm}^{2}$ ). The results were interpreted in terms of solvent evaporation and individual vehicle effects.

\section{EXPERIMENTAL}

\section{Chemicals}

${ }^{3} \mathrm{H}$-minoxidil (Minoxidil, [piperyl- $3,4-{ }^{3} \mathrm{H}$ ], ART$0711-250 \mu \mathrm{Ci}$ ) was purchased from American Radiolabeled Chemicals, Inc. (St. Louis, MO). Minoxidil was supplied by the Therapeutics Research Unit. Ethanol and PG were purchased from Sigma-Aldrich Pty. Ltd. (Sydney, Australia). The scintillation cocktail used was EmulsifierSafe (PerkinElmer Pty Ltd, Melbourne, Australia). For cyanoacrylate casting, we used "Quick Fix Supa Glue" (Selleys Pty Ltd, Padstow, Australia). Formulations containing $2 \%(\mathrm{~g} / \mathrm{mL})$ minoxidil and a trace amount of ${ }^{3} \mathrm{H}$-minoxidil (approx. $1 \mu \mathrm{Ci} / 7 \mu \mathrm{L}$ ) were prepared in three different vehicle mixtures. These contained (by volume) $60 \%$ ethanol, $20 \% \mathrm{PG}$, and $20 \%$ water (formulation 1); $80 \%$ ethanol and 20\% PG (formulation 2); and $80 \% \mathrm{PG}$ and $20 \%$ water (formulation 3). In the following, these formulations will be designated 60:20:20, 80:20:0, and $0: 80: 20$, respectively.

\section{Human Skin Membranes}

Human abdominal skin was obtained from volunteers undergoing elective surgery. The collection of skin was approved by the Princess Alexandra Hospital Research Committee (Approval no. 097/ 090, administered by the University of Queensland Human Ethics Committee) and donors gave informed written consent. Full thickness skin, which was used in the majority of experiments, was separated from underlying fat and epidermal and dermal membranes were prepared from full thickness skin by floating the skin in water at $60^{\circ} \mathrm{C}$ for $1 \mathrm{~min}$ and peeling off the epidermal layer. Both full thickness skin and epidermal membranes were stored frozen at $-20^{\circ} \mathrm{C}$ until use. 


\section{In Vitro Permeation Studies}

The experiments were performed using full thickness skin as described above, from three different donors (five replicates per skin) in glass horizontal static Franz-type diffusion cells, using phosphate buffered saline, $\mathrm{pH} 7.4$ (PBS) as the receptor phase. The exposed skin surface area was $1.33 \mathrm{~cm}^{2}$ in all cases. The diffusion cells were placed in a water bath, to maintain the receptor phase temperature at $35^{\circ} \mathrm{C}$, over a magnetic 15 point stirring plate. Magnetic stirrer bars in the bottom of each receptor chamber maintained mixing of the receptor fluid. The prepared Franz cells remained in the water bath for a minimum of 30 min before dosing, to ensure the skin was adequately hydrated. After hydration, resistance across the skin was measured with a standard multimeter, with PBS in the donor and receptor chambers. A resistance reading of $\geq 20 \mathrm{k} \Omega$ indicated acceptable skin integrity. If resistance was $<20 \mathrm{k} \Omega$, skin was replaced in that cell. The donor and receptor chambers were then emptied, the receptor chamber was refilled with fresh PBS and the cells equilibrated in the water bath for a further $15 \mathrm{~min}$ before dosing.

Finite doses of the above formulations were applied to the skin at the rate of $5 \mu \mathrm{L} / \mathrm{cm}^{2}$ (i.e., $7 \mu \mathrm{L}$ in all cases, for a $1.33 \mathrm{~cm}^{2}$ membrane area), using an M10 Gilson positive displacement pipette. The $7 \mu \mathrm{L}$ applications were spread evenly over the skin surface with the rubber tip of a $1 \mathrm{~mL}$ syringe plunger, using a gentle horizontal motion to prevent damage to the skin surface and the amount of radioactive material remaining on the rubber tip was quantitated, in order to ascertain the actual dose applied.

At appropriate times up to $\sim 28 \mathrm{~h}, 200 \mu \mathrm{L}$ samples were removed from the sampling arm of the diffusion cell using extra long pipette tips, with this volume being replaced with fresh receptor solution after each sample to maintain the receptor chamber volume.

\section{Differential Stripping Studies}

Separate Franz diffusion studies were carried out for $30 \mathrm{~min}, 2 \mathrm{~h}$ and $5 \mathrm{~h}$ as above, with skin from one donor and four replicates. After the designated time, the cells were dismantled and the skin was swabbed three times with an ethanol moistened cotton buds. These swab samples were saved separately for radioactivity quantitation. After 10 min air drying, the skin was subjected to tape stripping, which involves even application of adhesive tape to the exposed skin surface and careful removal, up to a maximum of 20 times. Individual tapes were placed into separate scintillation vials and soaked overnight with $2 \mathrm{~mL}$ scintillation fluid (Emulsifier-Safe) before vortexing and quantitation by liquid scintillation counting (LSC). The total amount of minoxidil deposited within the SC was obtained by summation of the amounts measured on the individual tapes. Results for the first two tapes were not included, as this represents unabsorbed surface material only.

Following tape stripping, follicular casts were prepared. One drop of superglue was placed on a glass microscope slide, which was then pressed onto the surface of the stripped skin and held in place under light pressure. After $15 \mathrm{~min}$, when the glue had hardened, the slide was peeled carefully from the skin, taking the contents of the skin appendages with it. The superglue was dissolved by rubbing with acetone-soaked cotton buds $(4 \times)$ and the individual cotton buds containing the sampled follicular contents were soaked overnight in $2 \mathrm{~mL}$ scintillation fluid before vortexing and quantitation by LSC. The processes of thorough swabbing and tape stripping of the skin ensured that all surface material was removed prior to cyanoacrylate casting, so that material recovered with the casts represented appendageal content.

\section{Data Analysis}

Skin penetration was assessed by the comparison of cumulative amount versus time profiles. Where appropriate, statistical differences were determined by the General Linear Model (multivariate), with post hoc comparisons by Tukey's test (SPSS ver. 14.0).

\section{RESULTS}

\section{Uptake of Minoxidil Into Follicles and Stratum Corneum}

The recovery of minoxidil from cyanoacrylate casts is shown in Figure 1A. Significantly more minoxidil was recovered from the appendages after application of formulations containing ethanol $(60: 20: 20$ and 80:20:0) than the formulation lacking ethanol $(0: 80: 20)$, particularly at early times of $30 \mathrm{~min}-2 \mathrm{~h}$ following application. The presence of water in the ethanol-containing formulations also appeared to lead to greater 

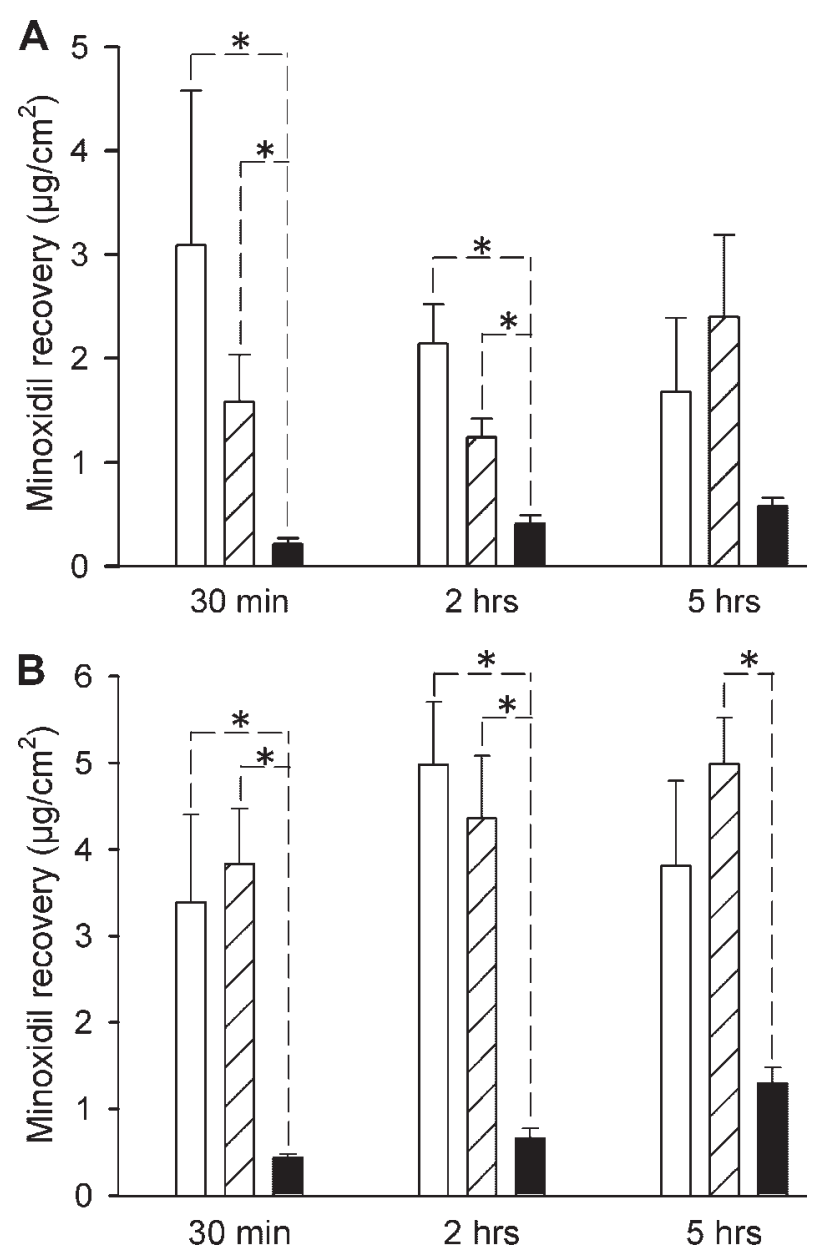

Figure 1. Minoxidil content of (A) follicular casts and (B) tape strips 3-20, taken from full thickness human skin after $30 \mathrm{~min}, 2 \mathrm{~h}$, and $5 \mathrm{~h}$ exposure to minoxidilcontaining formulations applied at a dose of $5 \mu \mathrm{L} / \mathrm{cm}^{2}$ (mean $\pm \mathrm{SE}, n=4$ for each formulation). Formulations contained ethanol, propylene glycol, and water in volume ratios of 60:20:20 (open bars), 80:20:0 (crosshatched bars), and 0:80:20 (filled bars). Significant differences $(p<0.05)$ between pairs of mean values are indicated by asterisks.

minoxidil recovery from casts at early times, as seen in the comparison of 60:20:20 and 80:20:0 in Figure 1A, although the difference between these formulations failed to reach statistical significance.

The effect of the different formulations on minoxidil penetration into the $\mathrm{SC}$ and the subsequent diffusion concentrations established within the membrane, assessed by tape stripping, is illustrated in Figure 1B. Application of the 60:20:20 and 80:20:0 formulations (containing ethanol), resulted in significantly more minoxidil being recovered from the SC than the 0:80:20 formulation (containing no ethanol). In contrast to results from cyanoacrylate casts, recovery from $\mathrm{SC}$ was not enhanced by the presence of water in the ethanol-containing formulations.

\section{Penetration Profile up to $28 \mathrm{~h}$}

The relative cumulative amounts of minoxidil penetrating human full thickness skin from each of the three test formulations are shown in Figure 2. As shown by the slopes of the penetration versus time curves, minoxidil flux in the first $12 \mathrm{~h}$ was in the order 60:20:20 > 80:20:0 $>0: 80: 20$. The highest flux was seen with the vehicle containing ethanol and water and the lowest flux was seen with the vehicle containing no ethanol. A dramatic change occurred in the subsequent $12 \mathrm{~h}$, with an accelerated flux seen with the 0:80:20 vehicle.

The amount of minoxidil retained in the skin at the end of the study (representing material that had passed through the SC barrier) was in the order 0:80:20>60:20:20>80:20:0, the same as observed for recovery of minoxidil from the receptor chamber. However, the amount of minoxidil retained in the SC, represented by recovery from the tape strips, showed a different pattern (60:20:20 $>0: 80: 20>80: 20: 0)$, supporting the notion that the 0:80:20 formulation allowed minoxidil to more readily traverse the SC barrier at longer times.

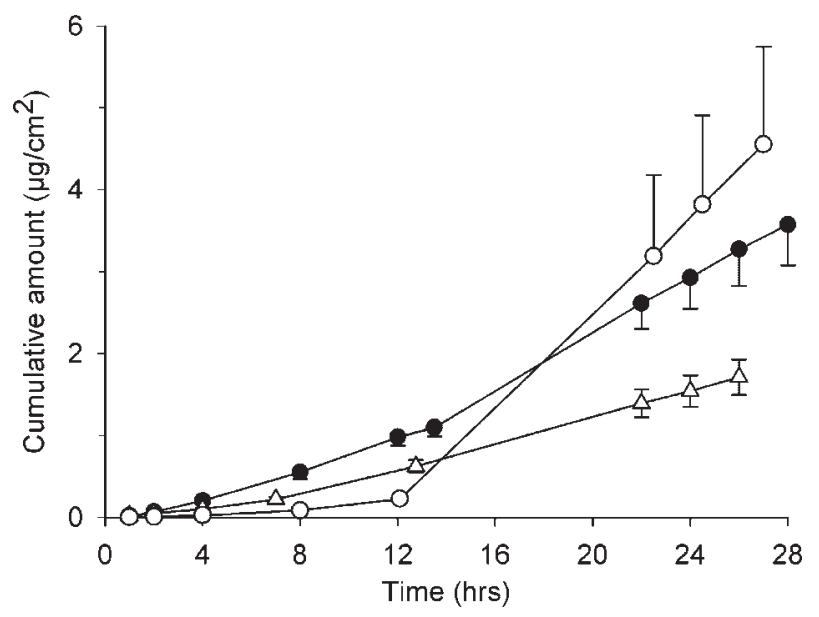

Figure 2. Mean $( \pm \mathrm{SE})$ cumulative minoxidil penetration versus time following application of $2 \%$ minoxidil formulations $\left(5 \mu \mathrm{L} / \mathrm{cm}^{2}\right)$. Formulations contained ethanol, propylene glycol, and water in volume ratios of 60:20:20 (closed circles), 80:20:0 (open triangles), and 0:80:20 (open circles). Data from three different human full thickness skins and five cells per skin were combined. 


\section{DISCUSSION}

Although it was previously thought that the appendageal route was relatively unimportant, as the area of skin containing these appendages is probably $<0.1 \%$ of the total skin area, ${ }^{4,18}$ we showed in this study that minoxidil recovery from appendages and SC was of the same order as the cumulative minoxidil penetration through the skin. In addition, we showed that the amount of minoxidil recovered from the appendages and SC after $30 \mathrm{~min}$ and $2 \mathrm{~h}$ exposure was dependent on the vehicle composition of the applied minoxidil formulation. The presence of ethanol and possibly water in the formulation promoted accumulation of minoxidil in these areas.

We also observed differences in the cumulative amount of minoxidil measured in the receptor fluid. At earlier times (before about $12 \mathrm{~h}$ exposure), the effect of formulation was similar to that seen on appendageal and SC recovery (i.e., the ethanol-containing formulations were favored). A change occurred after $12 \mathrm{~h}$, with a marked increase in flux seen with the formulation containing the greatest proportion of PG but no ethanol. These results may provide important mechanistic insights.

\section{Effects at Early Times}

Minoxidil was applied in three different vehicle mixtures, two of which contained ethanol and one which did not. We showed significantly greater amounts of minoxidil recovered from SC (by tape stripping) and from appendages (by cyanoacrylate casts) after penetration from ethanol-containing formulations (60:20:20 and 80:20:0), compared to the formulation lacking ethanol $(0: 80: 20)$. This was particularly apparent at early times (30 min and $2 \mathrm{~h}$ ). Similar effects by ethanol have been seen in previous studies. Tata et al. ${ }^{19}$ showed increasing recovery of minoxidil from hairless mouse epidermis and dermis with increasing ethanol in the vehicle, while Grams and Bouwstra ${ }^{9}$ found that follicular penetration of low lipophilicity fluorescent dyes was increased by addition of ethanol to the vehicle. The same ethanolcontaining formulations caused greater cumulative receptor penetration for the first $12 \mathrm{~h}$ of application. Early minoxidil uptake favoring ethanol-containing formulations probably reflects the partitioning of ethanol (in which minoxidil is approximately six times more soluble than in water) rapidly into lipid-rich skin compartments (including follicles), carrying minoxidil with it. Such a "pull effect" mechanism has been invoked to explain the enhancement of skin penetration of mefenamic acid by ethanol. ${ }^{20}$

In considering the effects at earlier times, it became clear that evaporation of the more volatile components, ethanol and water, was important. We hypothesized that the follicle, $\mathrm{SC}$, and receptor minoxidil content resulting from application of a particular formulation would be determined by the minoxidil concentration remaining on the skin after evaporation of the volatile solvents, ethanol, and water. An ethanol/PG/water mixture containing minoxidil would pass through a range of evaporation stages; viz. (i) ethanol and water evaporating but still present, (ii) ethanol and water completely evaporated but PG largely undepleted, (iii) PG evaporating, to finally leave any remaining minoxidil deposited on the skin surface. Previous investigations of these solvent mixtures found that after about $2 \mathrm{~h}$, ethanol and water had completely evaporated, while most of the PG remained. ${ }^{21}$ In the formulations studied here, the minoxidil concentration would therefore increase in proportion to the decrease in volume caused by evaporation.

After about $2 \mathrm{~h}$, in 60:20:20 or 80:20:0 mixtures of ethanol/PG/water, the minoxidil concentration would increase fivefold from $2 \%$ to $10 \%$ after evaporation of ethanol and water, but in a 0:80:20 mixture it would rise from $2 \%$ to only $2.5 \%$. At the $35^{\circ} \mathrm{C}$ temperatures at which the experiment was conducted, minoxidil would be expected to remain in solution at $10 \%$, without reaching supersaturation. As the flux of solute transport is a function of thermodynamic activity, which in turn is proportional to solute concentration in the vehicle, we would therefore expect that at this point, the instantaneous minoxidil flux for the 60:20:20 and 80:20:0 mixtures would be four times that seen with 0:80:20. This would be reflected in the amount of minoxidil recovered from receptor, skin, and appendages. At $5 \mathrm{~h}$, with only $\mathrm{PG}$ expected to remain, cast and tape amounts for the 60:20:20 and 80:20:0 mixtures were four times those seen with the 0:80:20 mixture, as predicted.

\section{Penetration Profile Over Longer Time $(28 \mathrm{~h})$}

An apparent abrupt change in penetration flux occurred for the 0:80:20 formulation only, at $\sim 12 \mathrm{~h}$. Significantly, these observations were seen 
consistently across the three separate skin samples used in the experiments. This suggests a clear change in mechanism of transport over time. In the initial $12 \mathrm{~h}$, maximum penetration was obtained with the formulations containing ethanol and the least $P G$, indicating that $P G$ is thermodynamically unfavourable for penetration. Importantly, for the two formulations with the same water content $(60: 20: 20$ and $0: 80: 20)$, the ratio of amount penetrated (4.7) was close to that of 4 , which would have been predicted on the basis of the concentration of minoxidil remaining in the residue $\mathrm{PG}$ on the skin $(5.6 \mu \mathrm{L}$ for $0: 80: 20,1.4 \mu \mathrm{L}$ for 60:20:20).

By contrast, after the initial $12 \mathrm{~h}$, maximum penetration was soon obtained by the formulation with the most PG. We interpret this as a change in transport mechanism brought about by a physical change in the membrane (SC) induced by PG. Recognizing that SC uptake represents mainly a partition process whereas skin penetration reflects both partition and diffusion, this may be due to the slow uptake of PG into the membrane and its gradual alteration of SC permeability. Interestingly, Tata et al. ${ }^{19}$ saw a similar enhancement of minoxidil uptake into hairless mouse epidermis after $12 \mathrm{~h}$ with $\mathrm{PG}$-rich vehicles. The relatively poor SC minoxidil uptake from the 80:20:0 formulation may reflect the low permeability of the dehydrated membrane in this water-free formulation.

\section{ACKNOWLEDGMENTS}

The authors are grateful for support from Pfizer Global Research and Development, Ann Arbor, MI and the National Health and Medical Research Council of Australia. Yuri Anissimov and Owen Jepps assisted in early and late discussions related to this work. Valuable technical support was provided by Ms. Jenny Ordonez, Ms. Jill Walton, and Ms. Ya-Ting Wu.

\section{REFERENCES}

1. Lademann J, Knorr F, Richter H, Blume-Peytavi U, Vogt A, Antoniou C, Sterry W, Patzelt A. Hair follicles-An efficient storage and penetration pathway for topically applied substances. Summary of recent results obtained at the Center of Experimental and Applied Cutaneous Physiology, Charite-Universitatsmedizin Berlin, Germany. Skin Pharmacol Physiol 2008. 21:150-155.
2. Frum Y, Bonner MC, Eccleston GM, Meidan VM. The influence of drug partition coefficient on follicular penetration: In vitro human skin studies. Eur J Pharm Sci 2007. 30:280-287.

3. Meidan VM, Bonner MC, Michniak BB. Transfollicular drug delivery-is it a reality? Int J Pharm 2005. 306:1-14.

4. Scheuplein RJ, Blank IH. Permeability of the skin. Physiol Rev 1971. 51:702-747.

5. Rougier A, Lotte C, Maibach HI. In vivo percutaneous penetration of some organic compounds related to anatomic site in humans: Predictive assessment by the stripping method. J Pharm Sci 1987. 76:451-454.

6. Hueber F, Wepierre J, Schaefer H. Role of transepidermal and transfollicular routes in percutaneous absorption of hydrocortisone and testosterone: In vivo study in the hairless rat. Skin Pharmacol 1992. 5:99-107.

7. Teichmann A, Otberg N, Jacobi U, Sterry W, Lademann J. Follicular penetration: Development of a method to block the follicles selectively against the penetration of topically applied substances. Skin Pharmacol Physiol 2006. 19:216-223.

8. El Maghraby GM, Williams AC, Barry BW. Skin hydration and possible shunt route penetration in controlled estradiol delivery from ultradeformable and standard liposomes. J Pharm Pharmacol 2001. 53:1311-1322.

9. Grams YY, Bouwstra JA. Penetration and distribution of three lipophilic probes in vitro in human skin focusing on the hair follicle. J Control Release 2002. 83:253-262.

10. Herkenne C, Alberti I, Naik A, Kalia YN, Mathy FX, Preat V, Guy RH. In vivo methods for the assessment of topical drug bioavailability. Pharm Res 2008. 25:87-103.

11. Teichmann A, Jacobi U, Ossadnik M, Richter H, Koch S, Sterry W, Lademann J. Differential stripping: Determination of the amount of topically applied substances penetrated into the hair follicles. J Invest Dermatol 2005. 125:264-269.

12. Marks R, Dawber RP. Skin surface biopsy: An improved technique for the examination of the horny layer. Br J Dermatol 1971. 84:117-123.

13. Williams AC, Barry BW. Penetration enhancers. Adv Drug Deliv Rev 2004. 56:603-618.

14. Kaushik D, Batheja P, Kilfoyle B, Rai V, MichniakKohn B. Percutaneous permeation modifiers: Enhancement versus retardation. Expert Opin Drug Deliv 2008. 5:517-529.

15. Trommer H, Neubert RH. Overcoming the stratum corneum: The modulation of skin penetration. A review. Skin Pharmacol Physiol 2006. 19:106-121.

16. Messenger AG, Rundegren J. Minoxidil: Mechanisms of action on hair growth. Br J Dermatol 2004. 150:186-194.

17. Shorter K, Farjo NP, Picksley SM, Randall VA. Human hair follicles contain two forms of ATP- 
sensitive potassium channels, only one of which is sensitive to minoxidil. FASEB J 2008. 22:1725-1736.

18. Barry BW. Novel mechanisms and devices to enable successful transdermal drug delivery. Eur J Pharm Sci 2001. 14:101-114.

19. Tata S, Weiner N, Flynn G. Relative influence of ethanol and propylene glycol cosolvents on deposition of minoxidil into the skin. J Pharm Sci 1994. 83:1508-1510.
20. Heard CM, Kung D, Thomas CP. Skin penetration enhancement of mefenamic acid by ethanol and 1,8-cineole can be explained by the "pull" effect. Int J Pharm 2006. 321:167-170.

21. Chiang CM, Flynn GL, Weiner ND, Szpunar GJ. Bioavailability assessment of topical delivery systems - Effect of vehicle evaporation upon in vitro delivery of minoxidil from solution formulations. Int J Pharm 1989. 55:229-236. 